\title{
ON HERMITE-HADAMARD TYPE INEQUALITIES FOR $F$-CONVEX FUNCTION
}

\author{
H. BUDAK, T. TUNÇ, AND M. Z. SARIKAYA
}

Received 27 October, 2017

\begin{abstract}
In this study, we firstly give some properties the family $F$ and $F$-convex function which are defined by B. Samet. Then, we obtain some midpoint inequalities for differentiable function. Moreover, we establish some midpoint and trapezoid type inequalities for function whose second derivatives in absolute value are $F$-convex.
\end{abstract}

2010 Mathematics Subject Classification: 26D07; 26D10; 26D15; 26A33

Keywords: Hermite-Hadamard inequality, $F$-convex, midpoint inequality, trapezoid inequality

\section{INTRODUCTION}

Let $f: I \subseteq R \rightarrow R$ be a convex function on the interval $I$ of real numbers and $a, b \in I$ with $a<b$. If $f$ is a convex function then the following double inequality, which is well known in the literature as the Hermite-Hadamard inequality, holds [8]

$$
f\left(\frac{a+b}{2}\right) \leq \frac{1}{b-a} \int_{a}^{b} f(x) d x \leq \frac{f(a)+f(b)}{2} .
$$

Note that some of the classical inequalities for means can be derived from (1.1) for appropriate particular selections of the mapping $f$. Both inequalities hold in the reversed direction if $\mathrm{f}$ is concave (1.1).

It is well known that the Hermite-Hadamard inequality plays an important role in nonlinear analysis. Over the last decade, this classical inequality has been improved and generalized in a number of ways; there have been a large number of research papers written on this subject, (see, $[3,4,6,9,12,13]$ ) and the references therein.

Over the years, many type of convexity have been defined, such as quasi-convex [1], pseudo-convex [7], strongly convex [10], $\varepsilon$-convex [2], $s$-convex [5], $h$-convex [14] and etc. Recently, Samet [11] have defined a new concept of convexity that depends on a certain function satisfying some axioms, that generalizes different types of convexity, including $\varepsilon$-convex functions, $\alpha$-convex functions, $h$-convex functions and many others.

Recall the family $\mathcal{F}$ of mappings $F: \mathbb{R} \times \mathbb{R} \times \mathbb{R} \times[0,1] \rightarrow \mathbb{R}$ satisfying the following axioms: 
(A1) If $u_{i} \in L^{1}(0,1), i=1,2,3$, then for every $\lambda \in[0,1]$, we have

$$
\int_{0}^{1} F\left(u_{1}(t), u_{2}(t), u_{3}(t), \lambda\right) d t=F\left(\int_{0}^{1} u_{1}(t) d t, \int_{0}^{1} u_{2}(t) d t, \int_{0}^{1} u_{3}(t) d t, \lambda\right) \text {. }
$$

(A2) For every $u \in L^{1}(0,1), w \in L^{\infty}(0,1)$ and $\left(z_{1}, z_{2}\right) \in \mathbb{R}^{2}$, we have

$$
\left.\int_{0}^{1} F\left(w(t) u(t), w(t) z_{1}, w(t) z_{2}, t\right) d t=T_{F, w}\left(\int_{0}^{1} w(t) u(t) d t, z_{1}, z_{2}\right)\right),
$$

where $T_{F, w}: \mathbb{R} \times \mathbb{R} \times \mathbb{R} \rightarrow \mathbb{R}$ is a function that depends on $(F, w)$, and it is nondecreasing with respect to the first variable.

(A3) For any $\left(w, u_{1}, u_{2}, u_{3}\right) \in \mathbb{R}^{4}, u_{4} \in[0,1]$, we have

$$
w F\left(u_{1}, u_{2}, u_{3}, u_{4}\right)=F\left(w u_{1}, w u_{2}, w u_{3}, u_{4}\right)+L_{w}
$$

where $L_{w} \in \mathbb{R}$ is a constant that depends only on $w$.

Definition 1. Let $f:[a, b] \rightarrow \mathbb{R},(a, b) \in \mathbb{R}^{2}, a<b$, be a given function. We say that $f$ is a convex function with respect to some $F \in \mathcal{F}$ (or $F$-convex function) iff

$$
F(f(t x+(1-t) y), f(x), f(y), t) \leq 0, \quad(x, y, t) \in[a, b] \times[a, b] \times[0,1] .
$$

Remark 1. 1) Let $\varepsilon \geq 0$, and let $f:[a, b] \rightarrow \mathbb{R},(a, b) \in \mathbb{R}^{2}, a<b$, be an $\varepsilon$-convex function, that is (see [2])

$$
f(t x+(1-t) y) \leq t f(x)+(1-t) f(y)+\varepsilon,(x, y, t) \in[a, b] \times[a, b] \times[0,1] .
$$

Define the functions $F: \mathbb{R} \times \mathbb{R} \times \mathbb{R} \times[0,1] \rightarrow \mathbb{R}$ by

$$
F\left(u_{1}, u_{2}, u_{3}, u_{4}\right)=u_{1}-u_{4} u_{2}-\left(1-u_{4}\right) u_{3}-\varepsilon
$$

and $T_{F, w}: \mathbb{R} \times \mathbb{R} \times \mathbb{R} \rightarrow \mathbb{R}$ by

$$
T_{F, w}\left(u_{1}, u_{2}, u_{3}\right)=u_{1}-\left(\int_{0}^{1} t w(t) d t\right) u_{2}-\left(\int_{0}^{1}(1-t) w(t) d t\right) u_{3}-\varepsilon .
$$

For

it is clear that $F \in \mathscr{F}$ and

$$
L_{w}=(1-w) \varepsilon
$$

$F(f(t x+(1-t) y), f(x), f(y), t)=f(t x+(1-t) y)-t f(x)-(1-t) f(y)-\varepsilon \leq 0$, that is $f$ is an $F$-convex function. Particularly, taking $\varepsilon=0$, we show that if $f$ is a convex function then $f$ is an $F$-convex function with respect to $F$ defined above.

2) Let $f:[a, b] \rightarrow \mathbb{R},(a, b) \in \mathbb{R}^{2}, a<b$, be an $\alpha$-convex function, $\alpha \in(0,1]$, that is

$$
f(t x+(1-t) y) \leq t^{\alpha} f(x)+\left(1-t^{\alpha}\right) f(y),(x, y, t) \in[a, b] \times[a, b] \times[0,1] .
$$


Define the functions $F: \mathbb{R} \times \mathbb{R} \times \mathbb{R} \times[0,1] \rightarrow \mathbb{R}$ by

$$
F\left(u_{1}, u_{2}, u_{3}, u_{4}\right)=u_{1}-u_{4}^{\alpha} u_{2}-\left(1-u_{4}^{\alpha}\right) u_{3}
$$

and $T_{F, w}: \mathbb{R} \times \mathbb{R} \times \mathbb{R} \rightarrow \mathbb{R}$ by

$$
T_{F, w}\left(u_{1}, u_{2}, u_{3}\right)=u_{1}-\left(\int_{0}^{1} t^{\alpha} w(t) d t\right) u_{2}-\left(\int_{0}^{1}\left(1-t^{\alpha}\right) w(t) d t\right) u_{3} .
$$

For $L_{w}=0$, it is clear that $F \in \mathscr{F}$ and

$$
F(f(t x+(1-t) y), f(x), f(y), t)=f(t x+(1-t) y)-t^{\alpha} f(x)-\left(1-t^{\alpha}\right) f(y) \leq 0,
$$

that is $f$ is an $F$-convex function.

3) Let $h: J \rightarrow[0, \infty)$ be a given function which is not identical to 0 , where $J$ is an interval in $\mathbb{R}$ such that $(0,1) \subseteq J$. Let $f:[a, b] \rightarrow[0, \infty),(a, b) \in \mathbb{R}^{2}, a<b$, be an $h$-convex function, that is (see [14])

$$
f(t x+(1-t) y) \leq h(t) f(x)+h(1-t) f(y),(x, y, t) \in[a, b] \times[a, b] \times[0,1] .
$$

Define the functions $F: \mathbb{R} \times \mathbb{R} \times \mathbb{R} \times[0,1] \rightarrow \mathbb{R}$ by

$$
F\left(u_{1}, u_{2}, u_{3}, u_{4}\right)=u_{1}-h\left(u_{4}\right) u_{2}-h\left(1-u_{4}\right) u_{3}
$$

and $T_{F, w}: \mathbb{R} \times \mathbb{R} \times \mathbb{R} \rightarrow \mathbb{R}$ by

$$
T_{F, w}\left(u_{1}, u_{2}, u_{3}\right)=u_{1}-\left(\int_{0}^{1} h(t) w(t) d t\right) u_{2}-\left(\int_{0}^{1} h(1-t) w(t) d t\right) u_{3} .
$$

For $L_{w}=0$, it is clear that $F \in \mathcal{F}$ and

$F(f(t x+(1-t) y), f(x), f(y), t)=f(t x+(1-t) y)-h(t) f(x)-h(1-t) f(y) \leq 0$, that is $f$ is an $F$-convex function.

In [11], author established the following Hermite-Hadamard type inequalities using the new convexity concept:

Theorem 1. Let $f:[a, b] \rightarrow \mathbb{R},(a, b) \in \mathbb{R}^{2}, a<b$, be an $F$-convex function, for some $F \in \mathcal{F}$. Suppose that $f \in L_{1}[a, b]$. Then

$$
\begin{gathered}
F\left(f\left(\frac{a+b}{2}\right), \frac{1}{b-a} \int_{a}^{b} f(x) d x, \frac{1}{b-a} \int_{a}^{b} f(x) d x, \frac{1}{2}\right) \leq 0 \\
T_{F, 1}\left(\frac{1}{b-a} \int_{a}^{b} f(x) d x, f(a), f(b)\right) \leq 0
\end{gathered}
$$


Theorem 2. Let $f: I^{\circ} \subseteq \mathbb{R} \rightarrow \mathbb{R}$ be a differentiable mapping on $I^{\circ},(a, b) \in$ $I^{\circ} \times I^{\circ}, a<b$. Suppose that

(i) $\left|f^{\prime}\right|$ is $F$-convex on $[a, b]$, for some $F \in \mathcal{F}$

(ii) the function $t \in(0,1) \rightarrow L_{w(t)}$ belongs to $L^{1}(0,1)$, where $w(t)=|1-2 t|$. Then,

$$
\begin{gathered}
T_{F, w}\left(\frac{2}{b-a}\left|\frac{f(a)+f(b)}{2}-\frac{1}{b-a} \int_{a}^{b} f(x) d x\right|,\left|f^{\prime}(a)\right|,\left|f^{\prime}(b)\right|\right) \\
+\int_{0}^{1} L_{w(t)} d t \leq 0 .
\end{gathered}
$$

Theorem 3. Let $f: I^{\circ} \subseteq \mathbb{R} \rightarrow \mathbb{R}$ be a differentiable mapping on $I^{\circ},(a, b) \in$ $I^{\circ} \times I^{\circ}, a<b$ and let $p>1$. Suppose that $\left|f^{\prime}\right|^{p /(p-1)}$ is $F$-convex on $[a, b]$, for some $F \in \mathcal{F}$ and $\left|f^{\prime}\right| \in L^{p /(p-1)}(a, b)$. Then

$$
T_{F, 1}\left(A(p, f),\left|f^{\prime}(a)\right|^{p /(p-1)},\left|f^{\prime}(b)\right|^{p /(p-1)}\right) \leq 0
$$

where

$$
A(p, f)=\left(\frac{2}{b-a}\right)^{\frac{p}{p-1}}(p+1)^{\frac{1}{p-1}}\left|\frac{f(a)+f(b)}{2}-\frac{1}{b-a} \int_{a}^{b} f(x) d x\right|^{\frac{p}{p-1}} .
$$

The following lemmas will be useful when we give our main result.

Lemma 1 ([6]). Let $f: I^{\circ} \subset \mathbb{R} \rightarrow \mathbb{R}$ be a differentiable mapping on $I^{\circ}, a, b \in I^{\circ}$ with $a<b$. If $f^{\prime} \in L^{1}[a, b]$, then we have

$$
\frac{1}{b-a} \int_{a}^{b} f(x) d x-f\left(\frac{a+b}{2}\right)=(b-a) \int_{0}^{1} p(t) f^{\prime}(t a+(1-t) b) d t,
$$

where

$$
p(t)= \begin{cases}t, & t \in\left[0, \frac{1}{2}\right] \\ t-1, & t \in\left(\frac{1}{2}, 1\right] .\end{cases}
$$

Lemma 2 ([4]). Let $f: I^{\circ} \subset \mathbb{R} \rightarrow \mathbb{R}$ be twice differentiable function on $I^{\circ}, a, b \in$ $I^{\circ}$ with $a<b$. If $f^{\prime \prime} \in L^{1}[a, b]$, then

$$
\frac{f(a)+f(b)}{2}-\frac{1}{b-a} \int_{a}^{b} f(x) d x=\frac{(b-a)^{2}}{2} \int_{0}^{1} t(1-t) f^{\prime \prime}(t a+(1-t) b) d t .
$$

Lemma 3 ([13] ). Let $f: I^{\circ} \subset \mathbb{R} \rightarrow \mathbb{R}$ be twice differentiable function on $I^{\circ}$, $a, b \in I^{\circ}$ with $a<b$. If $f^{\prime \prime} \in L^{1}[a, b]$, then

$$
\frac{1}{b-a} \int_{a}^{b} f(x) d x-f\left(\frac{a+b}{2}\right)
$$




$$
=\frac{(b-a)^{2}}{4} \int_{0}^{1} m(t)\left[f^{\prime \prime}(t a+(1-t) b)+f^{\prime \prime}(t b+(1-t) a)\right] d t,
$$

where

$$
m(t)= \begin{cases}t^{2}, & t \in\left[0, \frac{1}{2}\right] \\ (1-t)^{2}, & t \in\left(\frac{1}{2}, 1\right]\end{cases}
$$

\section{MidPoint TYPE INEQUALITIES FOR DIFFERENTIABLE FUNCTIONS}

In this section, we establish some midpoint type inequalities for functions whose derivatives absolute values are $F$-convex.

Theorem 4. Let $I \subseteq \mathbb{R}$ be an interval, $f: I^{\circ} \subseteq \mathbb{R} \rightarrow \mathbb{R}$ be a differentiable mapping on $I^{\circ}, a, b \in I^{\circ}, a<b$. Suppose that $\left|f^{\prime}\right|$ is $F$-convex on $[a, b]$, for some $F \in \mathcal{F}$ and the function $t \in[0,1] \rightarrow L_{w(t)}$ belongs to $L^{1}[0,1]$, where $w(t)=|p(t)|(p(t)$ is defined as in Lemma 1). Then,

$T_{F, w}\left(\frac{1}{b-a}\left|\frac{1}{b-a} \int_{a}^{b} f(x) d x-f\left(\frac{a+b}{2}\right)\right|,\left|f^{\prime}(a)\right|,\left|f^{\prime}(b)\right|\right)+\int_{0}^{1} L_{w(t)} d t \leq 0$.

Proof. Since $\left|f^{\prime}\right|$ is $F$-convex, we have

$$
F\left(\left|f^{\prime}(t a+(1-t) b)\right|,\left|f^{\prime}(a)\right|,\left|f^{\prime}(b)\right|, t\right) \leq 0, t \in[0,1] .
$$

Multiplying this inequality by $w(t)$ and using axiom (A3), we get

$$
F\left(w(t)\left|f^{\prime}(t a+(1-t) b)\right|, w(t)\left|f^{\prime}(a)\right|, w(t)\left|f^{\prime}(b)\right|, t\right)+L_{w(t)} \leq 0, t \in[0,1] .
$$

Integrating over $[0,1]$ with respect to the variable $t$ and using axiom (A2), we obtain

$$
T_{F, w}\left(\int_{0}^{1} w(t)\left|f^{\prime}(t a+(1-t) b)\right| d t,\left|f^{\prime}(a)\right|,\left|f^{\prime}(b)\right|\right)+\int_{0}^{1} L_{w(t)} d t \leq 0, t \in[0,1] .
$$

On the other hand using Lemma 1 we have

$$
\frac{1}{b-a}\left|\frac{1}{b-a} \int_{a}^{b} f(x) d x-f\left(\frac{a+b}{2}\right)\right| \leq \int_{0}^{1}|p(t)|\left|f^{\prime}(t a+(1-t) b)\right| d t .
$$

Since $T_{F, w}$ is nondecreasing with respect to the first variable, we get

$$
T_{F, w}\left(\frac{1}{b-a}\left|\frac{1}{b-a} \int_{a}^{b} f(x) d x-f\left(\frac{a+b}{2}\right)\right|,\left|f^{\prime}(a)\right|,\left|f^{\prime}(b)\right|\right)+\int_{0}^{1} L_{w(t)} d t \leq 0 .
$$

This completes the proof. 
Corollary 1. If $\left|f^{\prime}\right|$ is $\varepsilon$-convex on $[a, b], \varepsilon \geq 0$, then we have

$$
\left|\frac{1}{b-a} \int_{a}^{b} f(x) d x-f\left(\frac{a+b}{2}\right)\right| \leq(b-a)\left[\frac{\left|f^{\prime}(a)\right|+\left|f^{\prime}(b)\right|}{8}+\frac{\varepsilon}{4}\right] .
$$

Proof. It is known that an $\varepsilon$-convex is an $F$-convex. Using (1.4) with $w(t)=|p(t)|$, we obtain

$$
\int_{0}^{1} L_{w(t)} d t=\varepsilon \int_{0}^{1}(1-|p(t)|) d t=\frac{3}{4} \varepsilon
$$

From (1.3) with $w(t)=|p(t)|$, we get

$$
\begin{aligned}
T_{F, w}\left(u_{1}, u_{2}, u_{3}\right) & =u_{1}-\left(\int_{0}^{1} t|p(t)| d t\right) u_{2}-\left(\int_{0}^{1}(1-t)|p(t)| d t\right) u_{3}-\varepsilon \\
& =u_{1}-\frac{u_{2}+u_{3}}{8}-\varepsilon
\end{aligned}
$$

for $u_{1}, u_{2}, u_{3} \in \mathbb{R}$. Hence,

$$
\begin{aligned}
& T_{F, w}\left(\frac{1}{b-a}\left|\frac{1}{b-a} \int_{a}^{b} f(x) d x-f\left(\frac{a+b}{2}\right)\right|,\left|f^{\prime}(a)\right|,\left|f^{\prime}(b)\right|\right) \\
= & \frac{1}{b-a}\left|\frac{1}{b-a} \int_{a}^{b} f(x) d x-f\left(\frac{a+b}{2}\right)\right|-\frac{\left|f^{\prime}(a)\right|+\left|f^{\prime}(b)\right|}{8}-\varepsilon .
\end{aligned}
$$

Thus, by Theorem 4, we have

$$
\frac{1}{b-a}\left|\frac{1}{b-a} \int_{a}^{b} f(x) d x-f\left(\frac{a+b}{2}\right)\right|-\frac{\left|f^{\prime}(a)\right|+\left|f^{\prime}(b)\right|}{8}-\varepsilon+\frac{3}{4} \varepsilon \leq 0,
$$

that is

$$
\left|\frac{1}{b-a} \int_{a}^{b} f(x) d x-f\left(\frac{a+b}{2}\right)\right| \leq(b-a)\left[\frac{\left|f^{\prime}(a)\right|+\left|f^{\prime}(b)\right|}{8}+\frac{\varepsilon}{4}\right] .
$$

This completes the proof.

Remark 2. If we take $\varepsilon=0$ in Corollary 1 , then $\left|f^{\prime}\right|$ is convex and we have the inequality

$$
\left|\frac{1}{b-a} \int_{a}^{b} f(x) d x-f\left(\frac{a+b}{2}\right)\right| \leq(b-a)\left[\frac{\left|f^{\prime}(a)\right|+\left|f^{\prime}(b)\right|}{8}\right]
$$

which is given by [6]. 
Corollary 2. If $\left|f^{\prime}\right|$ is $\alpha$-convex on $[a, b], \alpha \in(0,1]$, then we have

$$
\begin{aligned}
\left|\frac{1}{b-a} \int_{a}^{b} f(x) d x-f\left(\frac{a+b}{2}\right)\right| \leq & (b-a)\left\{\frac{1}{(\alpha+1)(\alpha+2)}\left(1-\frac{1}{2^{\alpha+1}}\right)\left|f^{\prime}(a)\right|\right. \\
& \left.+\left[\frac{1}{4}-\frac{1}{(\alpha+1)(\alpha+2)}\left(1-\frac{1}{2^{\alpha+1}}\right)\right]\left|f^{\prime}(b)\right|\right\} .
\end{aligned}
$$

Proof. It is known that an $\alpha$-convex is an $F$-convex. Using (1.6) with $w(t)=$ $|p(t)|$, we obtain

$$
\begin{aligned}
T_{F, w}\left(u_{1}, u_{2}, u_{3}\right)= & u_{1}-\left(\int_{0}^{1} t^{\alpha}|p(t)| d t\right) u_{2}-\left(\int_{0}^{1}\left(1-t^{\alpha}\right)|p(t)| d t\right) u_{3} \\
= & u_{1}-\frac{1}{(\alpha+1)(\alpha+2)}\left(1-\frac{1}{2^{\alpha+1}}\right) u_{2} \\
& -\left[\frac{1}{4}-\frac{1}{(\alpha+1)(\alpha+2)}\left(1-\frac{1}{2^{\alpha+1}}\right)\right] u_{3}
\end{aligned}
$$

for $u_{1}, u_{2}, u_{3} \in \mathbb{R}$. Hence, by Theorem 4 , we have

$$
\begin{aligned}
& T_{F, w}\left(\frac{1}{b-a}\left|\frac{1}{b-a} \int_{a}^{b} f(x) d x-f\left(\frac{a+b}{2}\right)\right|,\left|f^{\prime}(a)\right|,\left|f^{\prime}(b)\right|\right) \\
= & \frac{1}{b-a}\left|\frac{1}{b-a} \int_{a}^{b} f(x) d x-f\left(\frac{a+b}{2}\right)\right| \\
& -\frac{1}{(\alpha+1)(\alpha+2)}\left(1-\frac{1}{2^{\alpha+1}}\right)\left|f^{\prime}(a)\right| \\
& -\left[\frac{1}{4}-\frac{1}{(\alpha+1)(\alpha+2)}\left(1-\frac{1}{2^{\alpha+1}}\right)\right]\left|f^{\prime}(b)\right| \leq 0,
\end{aligned}
$$

that is

$$
\begin{aligned}
\left|\frac{1}{b-a} \int_{a}^{b} f(x) d x-f\left(\frac{a+b}{2}\right)\right| \leq & (b-a)\left\{\frac{1}{(\alpha+1)(\alpha+2)}\left(1-\frac{1}{2^{\alpha+1}}\right)\left|f^{\prime}(a)\right|\right. \\
& \left.+\left[\frac{1}{4}-\frac{1}{(\alpha+1)(\alpha+2)}\left(1-\frac{1}{2^{\alpha+1}}\right)\right]\left|f^{\prime}(b)\right|\right\}
\end{aligned}
$$

which completes the proof.

Corollary 3. If $\left|f^{\prime}\right|$ is $h$-convex on $[a, b]$, then we have the inequalty

$$
\left|\frac{1}{b-a} \int_{a}^{b} f(x) d x-f\left(\frac{a+b}{2}\right)\right| \leq(b-a)\left(\int_{0}^{1} h(t)|p(t)| d t\right)\left[\left|f^{\prime}(a)\right|+\left|f^{\prime}(b)\right|\right] \text {. }
$$


Proof. It is known that an $h$-convex is an $F$-convex. From (1.8) with $w(t)=$ $|p(t)|$, we have

$$
\begin{aligned}
T_{F, w}\left(u_{1}, u_{2}, u_{3}\right) & =u_{1}-\left(\int_{0}^{1} h(t)|p(t)| d t\right) u_{2}-\left(\int_{0}^{1} h(1-t)|p(t)| d t\right) u_{3} \\
& =u_{1}-\left(\int_{0}^{1} h(t)|p(t)| d t\right) u_{2}-\left(\int_{0}^{1} h(1-t)|p(1-t)| d t\right) u_{3} \\
& =u_{1}-\left(\int_{0}^{1} h(t)|p(t)| d t\right)\left(u_{2}+u_{3}\right)
\end{aligned}
$$

for $u_{1}, u_{2}, u_{3} \in \mathbb{R}$. Then,

$$
\begin{aligned}
& T_{F, w}\left(\frac{1}{b-a}\left|\frac{1}{b-a} \int_{a}^{b} f(x) d x-f\left(\frac{a+b}{2}\right)\right|,\left|f^{\prime}(a)\right|,\left|f^{\prime}(b)\right|\right) \\
= & \frac{1}{b-a}\left|\frac{1}{b-a} \int_{a}^{b} f(x) d x-f\left(\frac{a+b}{2}\right)\right|-\left(\int_{0}^{1} h(t)|p(t)| d t\right)\left[\left|f^{\prime}(a)\right|+\left|f^{\prime}(b)\right|\right] .
\end{aligned}
$$

Thus, by Theorem 4, we get

$$
\begin{aligned}
& \frac{1}{b-a}\left|\frac{1}{b-a} \int_{a}^{b} f(x) d x-f\left(\frac{a+b}{2}\right)\right| \\
& -\left(\int_{0}^{1} h(t)|p(t)| d t\right)\left[\left|f^{\prime}(a)\right|+\left|f^{\prime}(b)\right|\right] \leq 0
\end{aligned}
$$

which completes the proof.

Theorem 5. Let $f: I^{\circ} \subseteq \mathbb{R} \rightarrow \mathbb{R}$ be a differentiable mapping on $I^{\circ}, a, b \in I^{\circ}$, $a<b$ and $p>1$. Suppose that $\left|f^{\prime}\right|^{p /(p-1)}$ is $F$-convex on $[a, b]$, for some $F \in \mathscr{F}$ and $\left|f^{\prime}\right| \in L^{p /(p-1)}(a, b)$. Then we have

$$
T_{F, 1}\left(A_{1}(f, p),\left|f^{\prime}(a)\right|^{p /(p-1)},\left|f^{\prime}(b)\right|^{p /(p-1)}\right) \leq 0
$$

where

$$
A_{1}(f, p)=\left(\frac{2}{b-a}\right)^{\frac{p}{p-1}}(p+1)^{\frac{1}{p-1}}\left|\frac{1}{b-a} \int_{a}^{b} f(x) d x-f\left(\frac{a+b}{2}\right)\right|^{\frac{p}{p-1}} .
$$


Proof. As $\left|f^{\prime}\right|^{p /(p-1)}$ is $F$-convex, we have

$F\left(\left|f^{\prime}(t a+(1-t) b)\right|^{p /(p-1)},\left|f^{\prime}(a)\right|^{p /(p-1)},\left|f^{\prime}(b)\right|^{p /(p-1)}, t\right) \leq 0, t \in[0,1]$.

Using axiom (A2) with $w(t)=1$, we get

$$
T_{F, 1}\left(\int_{0}^{1}\left|f^{\prime}(t a+(1-t) b)\right|^{p /(p-1)} d t,\left|f^{\prime}(a)\right|^{p /(p-1)},\left|f^{\prime}(b)\right|^{p /(p-1)}\right) \leq 0 .
$$

Using Lemma 1 and Hölder inequality, we obtain

$$
\begin{aligned}
& \left|\frac{1}{b-a} \int_{a}^{b} f(x) d x-f\left(\frac{a+b}{2}\right)\right| \\
= & (b-a) \int_{0}^{1}|p(t)|\left|f^{\prime}(t a+(1-t) b)\right| d t \\
\leq & (b-a)\left(\int_{0}^{1}|p(t)|^{p} d t\right)^{\frac{1}{p}}\left(\int_{0}^{1}\left|f^{\prime}(t a+(1-t) b)\right|^{p /(p-1)} d t\right)^{\frac{p-1}{p}} \\
= & \frac{b-a}{2}\left(\frac{1}{p+1}\right)^{\frac{1}{p}}\left(\int_{0}^{1}\left|f^{\prime}(t a+(1-t) b)\right|^{p /(p-1)} d t\right)^{\frac{p-1}{p}},
\end{aligned}
$$

that is

$$
A_{1}(f, p) \leq \int_{0}^{1}\left|f^{\prime}(t a+(1-t) b)\right|^{p /(p-1)} d t .
$$

Since $T_{F, 1}$ is nondecreasing with respect to the first variable, we can obtain the desired result easily.

Corollary 4. If $\left|f^{\prime}\right|^{p /(p-1)}$ is $\varepsilon$-convex on $[a, b], \varepsilon \geq 0$, then we have

$$
\begin{aligned}
& \left|\frac{1}{b-a} \int_{a}^{b} f(x) d x-f\left(\frac{a+b}{2}\right)\right| \\
\leq & \frac{b-a}{2(p+1)^{\frac{1}{p}}}\left[\frac{\left|f^{\prime}(a)\right|^{p /(p-1)}+\left|f^{\prime}(b)\right|^{p /(p-1)}}{2}+\varepsilon\right]^{\frac{p-1}{p}} .
\end{aligned}
$$

Proof. Using (1.3) with $w(t)=1$, we have

$$
T_{F, 1}\left(u_{1}, u_{2}, u_{3}\right)=u_{1}-\frac{u_{2}+u_{3}}{2}-\varepsilon
$$

for $u_{1}, u_{2}, u_{3} \in \mathbb{R}$. Then, by the Theorem 5 , we have

$$
0 \geq T_{F, 1}\left(A_{1}(f, p),\left|f^{\prime}(a)\right|^{p /(p-1)},\left|f^{\prime}(b)\right|^{p /(p-1)}\right)
$$




$$
\begin{aligned}
= & A_{1}(f, p)-\frac{\left|f^{\prime}(a)\right|^{p /(p-1)}+\left|f^{\prime}(b)\right|^{p /(p-1)}}{2}-\varepsilon \\
= & \left(\frac{2}{b-a}\right)^{\frac{p}{p-1}}(p+1)^{\frac{1}{p-1}}\left|\frac{1}{b-a} \int_{a}^{b} f(x) d x-f\left(\frac{a+b}{2}\right)\right|^{\frac{p}{p-1}} \\
& -\frac{\left|f^{\prime}(a)\right|^{p /(p-1)}+\left|f^{\prime}(b)\right|^{p /(p-1)}}{2}-\varepsilon
\end{aligned}
$$

that is,

$$
\begin{aligned}
& \left|\frac{1}{b-a} \int_{a}^{b} f(x) d x-f\left(\frac{a+b}{2}\right)\right| \\
\leq & \frac{b-a}{2(p+1)^{\frac{1}{p}}}\left[\frac{\left|f^{\prime}(a)\right|^{p /(p-1)}+\left|f^{\prime}(b)\right|^{p /(p-1)}}{2}+\varepsilon\right]^{\frac{p-1}{p}} .
\end{aligned}
$$

Remark 3. If we choose $\varepsilon=0$ in Corollary 4 , then $\left|f^{\prime}\right|^{p /(p-1)}$ is convex and we have the inequality

$$
\begin{gathered}
\left|\frac{1}{b-a} \int_{a}^{b} f(x) d x-f\left(\frac{a+b}{2}\right)\right| \\
\leq \frac{b-a}{2(p+1)^{\frac{1}{p}}}\left[\frac{\left|f^{\prime}(a)\right|^{p /(p-1)}+\left|f^{\prime}(b)\right|^{p /(p-1)}}{2}\right]^{\frac{p-1}{p}} .
\end{gathered}
$$

Corollary 5. If $\left|f^{\prime}\right|^{p /(p-1)}$ is $\alpha$-convex, $\alpha \in(0,1]$, then we have the following inequality

$$
\begin{gathered}
\left|\frac{1}{b-a} \int_{a}^{b} f(x) d x-f\left(\frac{a+b}{2}\right)\right| \\
\leq \frac{b-a}{2(p+1)^{\frac{1}{p}}}\left[\frac{\left|f^{\prime}(a)\right|^{p /(p-1)}+\alpha\left|f^{\prime}(b)\right|^{p /(p-1)}}{\alpha+1}\right]^{\frac{p-1}{p}} .
\end{gathered}
$$

Proof. From (1.6) with $w(t)=1$, we have

$$
\begin{aligned}
T_{F, 1}\left(u_{1}, u_{2}, u_{3}\right) & =u_{1}-\left(\int_{0}^{1} t^{\alpha} d t\right) u_{2}-\left(\int_{0}^{1}\left(1-t^{\alpha}\right) d t\right) u_{3} \\
& =u_{1}-\frac{u_{2}}{\alpha+1}-\frac{\alpha}{\alpha+1} u_{3}
\end{aligned}
$$


for $u_{1}, u_{2}, u_{3} \in \mathbb{R}$. By the Theorem 5 , it follows that

$$
\begin{aligned}
0 \geq & T_{F, 1}\left(A_{1}(f, p),\left|f^{\prime}(a)\right|^{p /(p-1)},\left|f^{\prime}(b)\right|^{p /(p-1)}\right) \\
= & A_{1}(f, p)-\frac{\left|f^{\prime}(a)\right|^{p /(p-1)}}{\alpha+1}-\frac{\alpha}{\alpha+1}\left|f^{\prime}(b)\right|^{p /(p-1)} \\
= & \left(\frac{2}{b-a}\right)^{\frac{p}{p-1}}(p+1)^{\frac{1}{p-1}}\left|\frac{1}{b-a} \int_{a}^{b} f(x) d x-f\left(\frac{a+b}{2}\right)\right|^{\frac{p}{p-1}} \\
& -\frac{\left|f^{\prime}(a)\right|^{p /(p-1)}}{\alpha+1}-\frac{\alpha}{\alpha+1}\left|f^{\prime}(b)\right|^{p /(p-1)}
\end{aligned}
$$

which achieves the proof.

Corollary 6. If $\left|f^{\prime}\right|^{p /(p-1)}$ is $h$-convex, then we have the following inequality

$$
\begin{aligned}
& \left|\frac{1}{b-a} \int_{a}^{b} f(x) d x-f\left(\frac{a+b}{2}\right)\right| \\
\leq & \frac{b-a}{2(p+1)^{\frac{1}{p}}}\left(\int_{0}^{1} h(t) d t\right)^{\frac{p-1}{p}}\left[\left|f^{\prime}(a)\right|^{p /(p-1)}+\left|f^{\prime}(b)\right|^{p /(p-1)}\right]^{\frac{p-1}{p}} .
\end{aligned}
$$

Proof. Using (1.8) with $w(t)=1$, we have

$$
\begin{aligned}
T_{F, 1}\left(u_{1}, u_{2}, u_{3}\right) & =u_{1}-\left(\int_{0}^{1} h(t) d t\right) u_{2}-\left(\int_{0}^{1} h(1-t) d t\right) u_{3} \\
& =u_{1}-\left(\int_{0}^{1} h(t) d t\right)\left(u_{2}+u_{3}\right)
\end{aligned}
$$

for $u_{1}, u_{2}, u_{3} \in \mathbb{R}$. By the Theorem 5 , we obtain

$$
\begin{aligned}
0 & \geq T_{F, 1}\left(A_{1}(f, p),\left|f^{\prime}(a)\right|^{p /(p-1)},\left|f^{\prime}(b)\right|^{p /(p-1)}\right) \\
& =A_{1}(f, p)-\left(\int_{0}^{1} h(t) d t\right)\left(\left|f^{\prime}(a)\right|^{p /(p-1)}+\left|f^{\prime}(b)\right|^{p /(p-1)}\right) \\
& =\left(\frac{2}{b-a}\right)^{\frac{p}{p-1}}(p+1)^{\frac{1}{p-1}}\left|\frac{1}{b-a} \int_{a}^{b} f(x) d x-f\left(\frac{a+b}{2}\right)\right|^{\frac{p}{p-1}}
\end{aligned}
$$




$$
-\left(\int_{0}^{1} h(t) d t\right)\left(\left|f^{\prime}(a)\right|^{p /(p-1)}+\left|f^{\prime}(b)\right|^{p /(p-1)}\right) .
$$

This completes the proof.

\section{TRAPEZOID TYPE INEQUALITIES FOR TWICE DIFFERENTIABLE FUNCTIONS}

In this section, we obtain some trapezoid type inequalities for functions whose second derivatives absolute values are $F$-convex.

Theorem 6. Let $I \subseteq \mathbb{R}$ be an interval, $f: I^{\circ} \subseteq \mathbb{R} \rightarrow \mathbb{R}$ be a twice differentiable mapping on $I^{\circ},(a, b) \in I^{\circ} \times I^{\circ}, a<b$. Suppose that $\left|f^{\prime \prime}\right|$ is $F$-convex on $[a, b]$, for some $F \in \mathcal{F}$ and the function $t \in[0,1] \rightarrow L_{w(t)}$ belongs to $L^{1}[0,1]$, where $w(t)=$ $t(1-t)$. Then we have the following inequality

$$
\begin{gathered}
T_{F, w}\left(\frac{2}{(b-a)^{2}}\left|\frac{f(a)+f(b)}{2}-\frac{1}{b-a} \int_{a}^{b} f(x) d x\right|,\left|f^{\prime \prime}(a)\right|,\left|f^{\prime \prime}(b)\right|\right) \\
+\int_{0}^{1} L_{w(t)} d t \leq 0 .
\end{gathered}
$$

Proof. Since $\left|f^{\prime \prime}\right|$ is $F$-convex, we have

$$
F\left(\left|f^{\prime \prime}(t a+(1-t) b)\right|,\left|f^{\prime \prime}(a)\right|,\left|f^{\prime \prime}(b)\right|, t\right) \leq 0, t \in[0,1] .
$$

Multiplying this inequality by $w(t)$ and using axiom (A3), we get

$F\left(w(t)\left|f^{\prime \prime}(t a+(1-t) b)\right|, w(t)\left|f^{\prime \prime}(a)\right|, w(t)\left|f^{\prime \prime}(b)\right|, t\right)+L_{w(t)} \leq 0, t \in[0,1]$.

Integrating over $(0,1)$ with respect to the variable $t$ and using axiom (A2), we obtain

$$
T_{F, w}\left(\int_{0}^{1} w(t)\left|f^{\prime \prime}(t a+(1-t) b)\right| d t,\left|f^{\prime \prime}(a)\right|,\left|f^{\prime \prime}(b)\right|\right)+\int_{0}^{1} L_{w(t)} d t \leq 0, t \in[0,1] .
$$

On the other hand using Lemma 2, we have

$$
\frac{2}{(b-a)^{2}}\left|\frac{f(a)+f(b)}{2}-\frac{1}{b-a} \int_{a}^{b} f(x) d x\right| \leq \int_{0}^{1} t(1-t)\left|f^{\prime \prime}(t a+(1-t) b)\right| d t .
$$

Since $T_{F, w}$ is nondecreasing with respect to the first variable, we get

$$
\begin{gathered}
T_{F, w}\left(\frac{2}{(b-a)^{2}}\left|\frac{f(a)+f(b)}{2}-\frac{1}{b-a} \int_{a}^{b} f(x) d x\right|,\left|f^{\prime \prime}(a)\right|,\left|f^{\prime \prime}(b)\right|\right) \\
+\int_{0}^{1} L_{w(t)} d t \leq 0 .
\end{gathered}
$$


This completes the proof.

Corollary 7. Let $f: I^{\circ} \subset \mathbb{R} \rightarrow \mathbb{R}$ be twice differentiable function on $I^{\circ}, a, b \in I^{\circ}$ with $a<b$. Suppose that the function $\left|f^{\prime \prime}\right|$ is $\varepsilon$-convex on $[a, b], \varepsilon \geq 0$. Then we have

$$
\left|\frac{f(a)+f(b)}{2}-\frac{1}{b-a} \int_{a}^{b} f(x) d x\right| \leq \frac{(b-a)^{2}}{2}\left[\frac{\left|f^{\prime \prime}(a)\right|+\left|f^{\prime \prime}(b)\right|}{12}+\frac{\varepsilon}{6}\right] .
$$

Proof. We know that an $\varepsilon$-convex is an $F$-convex. Using (1.4) with $w(t)=$ $t(1-t)$, we obtain

$$
\int_{0}^{1} L_{w(t)} d t=\varepsilon \int_{0}^{1}(1-w(t)) d t=\frac{5 \varepsilon}{6} .
$$

From (1.3) with $w(t)=t(1-t)$, we get

$$
\begin{aligned}
T_{F, w}\left(u_{1}, u_{2}, u_{3}\right) & =u_{1}-\left(\int_{0}^{1} t^{2}(1-t) d t\right) u_{2}-\left(\int_{0}^{1} t(1-t)^{2} d t\right) u_{3}-\varepsilon \\
& =u_{1}-\frac{u_{2}+u_{3}}{12}-\varepsilon
\end{aligned}
$$

for $u_{1}, u_{2}, u_{3} \in \mathbb{R}$. Hence,

$$
\begin{aligned}
& T_{F, w}\left(\frac{2}{(b-a)^{2}}\left|\frac{f(a)+f(b)}{2}-\frac{1}{b-a} \int_{a}^{b} f(x) d x\right|,\left|f^{\prime \prime}(a)\right|,\left|f^{\prime \prime}(b)\right|\right) \\
= & \frac{2}{(b-a)^{2}}\left|\frac{f(a)+f(b)}{2}-\frac{1}{b-a} \int_{a}^{b} f(x) d x\right|-\frac{\left|f^{\prime \prime}(a)\right|+\left|f^{\prime \prime}(b)\right|}{12}-\varepsilon .
\end{aligned}
$$

Thus, by Theorem 6, we have

$$
\frac{2}{(b-a)^{2}}\left|\frac{f(a)+f(b)}{2}-\frac{1}{b-a} \int_{a}^{b} f(x) d x\right|-\frac{\left|f^{\prime \prime}(a)\right|+\left|f^{\prime \prime}(b)\right|}{12}-\varepsilon+\frac{5 \varepsilon}{6} \leq 0 .
$$

This completes the proof.

Remark 4. If we taking $\varepsilon=0$ in Corollary 7, then $\left|f^{\prime \prime}\right|$ is convex and we have the inequality

$$
\left|\frac{f(a)+f(b)}{2}-\frac{1}{b-a} \int_{a}^{b} f(x) d x\right| \leq \frac{(b-a)^{2}}{12}\left[\frac{\left|f^{\prime \prime}(a)\right|+\left|f^{\prime \prime}(b)\right|}{2}\right] .
$$

Corollary 8. Let $f: I^{\circ} \subset \mathbb{R} \rightarrow \mathbb{R}$ be twice differentiable function on $I^{\circ}, a, b \in I^{\circ}$ with $a<b$. Suppose that the function $\left|f^{\prime \prime}\right|$ is $\alpha$-convex on $[a, b], \alpha \in(0,1]$. Then we 
have

$$
\begin{aligned}
& \left|\frac{f(a)+f(b)}{2}-\frac{1}{b-a} \int_{a}^{b} f(x) d x\right| \\
\leq & \frac{(b-a)^{2}}{2}\left[\frac{\left|f^{\prime \prime}(a)\right|}{(\alpha+2)(\alpha+3)}-\left(\frac{1}{6}-\frac{1}{(\alpha+2)(\alpha+3)}\right)\left|f^{\prime \prime}(b)\right|\right] .
\end{aligned}
$$

Proof. We know that an $\alpha$-convex is an $F$-convex. From (1.6) with $w(t)=$ $t(1-t)$, we have

$$
\begin{aligned}
T_{F, w}\left(u_{1}, u_{2}, u_{3}\right) & =u_{1}-\left(\int_{0}^{1} t^{\alpha+1}(1-t) d t\right) u_{2}-\left(\int_{0}^{1}\left(1-t^{\alpha}\right) t(1-t) d t\right) u_{3} \\
& =u_{1}-\frac{u_{2}}{(\alpha+2)(\alpha+3)}-\left[\frac{1}{6}-\frac{1}{(\alpha+2)(\alpha+3)}\right] u_{3}
\end{aligned}
$$

for $u_{1}, u_{2}, u_{3} \in \mathbb{R}$. It follows that

$$
\begin{aligned}
& T_{F, w}\left(\frac{2}{(b-a)^{2}}\left|\frac{f(a)+f(b)}{2}-\frac{1}{b-a} \int_{a}^{b} f(x) d x\right|,\left|f^{\prime \prime}(a)\right|,\left|f^{\prime \prime}(b)\right|\right) \\
= & \frac{2}{(b-a)^{2}}\left|\frac{f(a)+f(b)}{2}-\frac{1}{b-a} \int_{a}^{b} f(x) d x\right| \\
& -\frac{\left|f^{\prime \prime}(a)\right|}{(\alpha+2)(\alpha+3)}-\left[\frac{1}{6}-\frac{1}{(\alpha+2)(\alpha+3)}\right]\left|f^{\prime \prime}(b)\right| .
\end{aligned}
$$

Consequently, by Theorem 6 , we obtain

$$
\begin{aligned}
& \frac{2}{(b-a)^{2}}\left|\frac{f(a)+f(b)}{2}-\frac{1}{b-a} \int_{a}^{b} f(x) d x\right| \\
& -\frac{\left|f^{\prime \prime}(a)\right|}{(\alpha+2)(\alpha+3)}-\left[\frac{1}{6}-\frac{1}{(\alpha+2)(\alpha+3)}\right]\left|f^{\prime \prime}(b)\right| \leq 0,
\end{aligned}
$$

which completes the proof.

Corollary 9. Let $f: I^{\circ} \subset \mathbb{R} \rightarrow \mathbb{R}$ be twice differentiable function on $I^{\circ}, a, b \in I^{\circ}$ with $a<b$. Suppose that the function $\left|f^{\prime \prime}\right|$ is $h-$ convex on $[a, b]$. Then we have

$$
\begin{aligned}
& \left|\frac{f(a)+f(b)}{2}-\frac{1}{b-a} \int_{a}^{b} f(x) d x\right| \\
\leq & (b-a)^{2}\left(\int_{0}^{1} h(t) t(1-t) d t\right)\left[\frac{\left|f^{\prime \prime}(a)\right|+\left|f^{\prime \prime}(b)\right|}{2}\right] .
\end{aligned}
$$


Proof. We know that an $h$-convex is an $F$-convex. From (1.8) with $w(t)=$ $t(1-t)$, we have

$$
\begin{aligned}
T_{F, w}\left(u_{1}, u_{2}, u_{3}\right) & =u_{1}-\left(\int_{0}^{1} h(t) w(t) d t\right) u_{2}-\left(\int_{0}^{1} h(1-t) w(t) d t\right) u_{3} \\
& =u_{1}-\left(\int_{0}^{1} h(t) t(1-t) d t\right) u_{2}-\left(\int_{0}^{1} h(1-t) t(1-t) d t\right) u_{3} \\
& =u_{1}-\left(\int_{0}^{1} h(t) t(1-t) d t\right)\left(u_{2}+u_{3}\right)
\end{aligned}
$$

for $u_{1}, u_{2}, u_{3} \in \mathbb{R}$. Then,

$$
\begin{aligned}
& T_{F, w}\left(\frac{2}{(b-a)^{2}}\left|\frac{f(a)+f(b)}{2}-\frac{1}{b-a} \int_{a}^{b} f(x) d x\right|,\left|f^{\prime \prime}(a)\right|,\left|f^{\prime \prime}(b)\right|\right) \\
= & \frac{2}{(b-a)^{2}}\left|\frac{f(a)+f(b)}{2}-\frac{1}{b-a} \int_{a}^{b} f(x) d x\right| \\
& -\left(\int_{0}^{1} h(t) t(1-t) d t\right)\left[\left|f^{\prime \prime}(a)\right|+\left|f^{\prime \prime}(b)\right|\right] .
\end{aligned}
$$

Thus, by Theorem 6, we get

$$
\begin{aligned}
= & \frac{2}{(b-a)^{2}}\left|\frac{f(a)+f(b)}{2}-\frac{1}{b-a} \int_{a}^{b} f(x) d x\right| \\
& -\left(\int_{0}^{1} h(t) t(1-t) d t\right)\left[\left|f^{\prime \prime}(a)\right|+\left|f^{\prime \prime}(b)\right|\right] \leq 0 .
\end{aligned}
$$

This finishes the proof.

Theorem 7. Let $f: I^{\circ} \subseteq \mathbb{R} \rightarrow \mathbb{R}$ be a twice differentiable mapping on $I^{\circ}, a, b \in$ $I^{\circ}, a<b$ and let $p>1$. Suppose that $\left|f^{\prime \prime}\right|^{p /(p-1)}$ is $F$-convex on $[a, b]$, for some $F \in \mathcal{F}$ and $\left|f^{\prime \prime}\right| \in L^{p /(p-1)}(a, b)$. Then we have

$$
T_{F, 1}\left(A_{2}(f, p),\left|f^{\prime \prime}(a)\right|^{p /(p-1)},\left|f^{\prime \prime}(b)\right|^{p /(p-1)}\right) \leq 0
$$

where

$$
A_{2}(f, p)=
$$




$$
\left(\frac{2}{(b-a)^{2}}\right)^{\frac{p}{p-1}}\left(\frac{1}{B(p+1, p+1)}\right)^{\frac{1}{p-1}}\left|\frac{f(a)+f(b)}{2}-\frac{1}{b-a} \int_{a}^{b} f(x) d x\right|^{\frac{p}{p-1}}
$$

and $B(x, y)$ is Euler beta function defined by

$$
B(x, y)=\int_{0}^{1} t^{x-1}(1-t)^{y-1} d t .
$$

Proof. Since $\left|f^{\prime \prime}\right|^{p /(p-1)}$ is $F$-convex, we have

$$
F\left(\left|f^{\prime \prime}(t a+(1-t) b)\right|^{p /(p-1)},\left|f^{\prime \prime}(a)\right|^{p /(p-1)},\left|f^{\prime \prime}(b)\right|^{p /(p-1)}, t\right) \leq 0, t \in[0,1] .
$$

Using axiom (A2) with $w(t)=1$, we get

$$
T_{F, 1}\left(\int_{0}^{1}\left|f^{\prime \prime}(t a+(1-t) b)\right|^{p /(p-1)} d t,\left|f^{\prime \prime}(a)\right|^{p /(p-1)},\left|f^{\prime \prime}(b)\right|^{p /(p-1)}\right) \leq 0 .
$$

Using Lemma 2 and Hölder inequality, we obtain

$$
\begin{aligned}
& \left|\frac{f(a)+f(b)}{2}-\frac{1}{b-a} \int_{a}^{b} f(x) d x\right| \\
= & \frac{(b-a)^{2}}{2} \int_{0}^{1}|t(1-t)|\left|f^{\prime \prime}(t a+(1-t) b)\right| d t \\
\leq & \frac{(b-a)^{2}}{2}\left(\int_{0}^{1} t^{p}(1-t)^{p} d t\right)^{\frac{1}{p}}\left(\int_{0}^{1}\left|f^{\prime \prime}(t a+(1-t) b)\right|^{p /(p-1)} d t\right)^{\frac{p-1}{p}} \\
= & \frac{(b-a)^{2}}{2}(B(p+1, p+1))^{\frac{1}{p}}\left(\int_{0}^{1}\left|f^{\prime \prime}(t a+(1-t) b)\right|^{p /(p-1)} d t\right)^{\frac{p-1}{p}},
\end{aligned}
$$

that is

$$
A_{2}(f, p) \leq \int_{0}^{1}\left|f^{\prime \prime}(t a+(1-t) b)\right|^{p /(p-1)} d t .
$$

Since $T_{F, 1}$ is nondecreasing with respect to the first variable, we have

$$
T_{F, 1}\left(A_{2}(f, p),\left|f^{\prime \prime}(a)\right|^{p /(p-1)},\left|f^{\prime \prime}(b)\right|^{p /(p-1)}\right) \leq 0 .
$$

Corollary 10. If $\left|f^{\prime \prime}\right|^{p /(p-1)}$ is $\varepsilon$-convex, we have the following inequality

$$
\left|\frac{f(a)+f(b)}{2}-\frac{1}{b-a} \int_{a}^{b} f(x) d x\right|
$$




$$
\leq \frac{(b-a)^{2}}{2}(B(p+1, p+1))^{\frac{1}{p}}\left[\frac{\left|f^{\prime \prime}(a)\right|^{p /(p-1)}+\left|f^{\prime \prime}(b)\right|^{p /(p-1)}}{2}+\varepsilon\right]^{\frac{p-1}{p}} .
$$

Proof. Using (2.1) and by Theorem 7, it can be proved easily. It is omitted.

Remark 5. If we choose $\varepsilon=0$ in Corollary 10, then we have

$$
\begin{aligned}
& \left|\frac{f(a)+f(b)}{2}-\frac{1}{b-a} \int_{a}^{b} f(x) d x\right| \\
\leq & \frac{(b-a)^{2}}{2}(B(p+1, p+1))^{\frac{1}{p}}\left[\frac{\left|f^{\prime \prime}(a)\right|^{p /(p-1)}+\left|f^{\prime \prime}(b)\right|^{p /(p-1)}}{2}\right]^{\frac{p-1}{p}} .
\end{aligned}
$$

Corollary 11. If $\left|f^{\prime \prime}\right|^{p /(p-1)}$ is $\alpha$-convex, we have the following inequality

$$
\begin{aligned}
& \left|\frac{f(a)+f(b)}{2}-\frac{1}{b-a} \int_{a}^{b} f(x) d x\right| \\
\leq & \frac{(b-a)^{2}}{2}(B(p+1, p+1))^{\frac{1}{p}}\left[\frac{\left|f^{\prime}(a)\right|^{p /(p-1)}+\alpha\left|f^{\prime}(b)\right|^{p /(p-1)}}{\alpha+1}\right]^{\frac{p-1}{p}} .
\end{aligned}
$$

Proof. Using (2.2) and by Theorem 7, it can be proved easily. It is omitted.

Corollary 12. If $\left|f^{\prime \prime}\right|^{p /(p-1)}$ is $h$-convex, we have the following inequality

$$
\begin{gathered}
\left|\frac{f(a)+f(b)}{2}-\frac{1}{b-a} \int_{a}^{b} f(x) d x\right| \\
\leq \frac{(b-a)^{2}}{2}(B(p+1, p+1))^{\frac{1}{p}}\left(\int_{0}^{1} h(t) d t\right)^{\frac{p-1}{p}} \\
{\left[\left|f^{\prime}(a)\right|^{p /(p-1)}+\left|f^{\prime}(b)\right|^{p /(p-1)}\right]^{\frac{p-1}{p}} .}
\end{gathered}
$$

Proof. Using (2.3) and by Theorem 7, it can be proved easily. It is omitted.

\section{Midpoint Type INEQUALITIES FOR TWICE DIFFERENTIABLE FUnCTIONS}

In this section, we prove some midpoint type inequalities for functions whose second derivatives absolute values are $F$-convex.

Theorem 8. Let $I \subseteq \mathbb{R}$ be an interval, $f: I^{\circ} \subseteq \mathbb{R} \rightarrow \mathbb{R}$ be a twice differentiable mapping on $I^{\circ},(a, b) \in I^{\circ} \times I^{\circ}, a<b$. Suppose that $\left|f^{\prime \prime}\right|$ is $F$-convex on $[a, b]$, 
for some $F \in \mathcal{F}$ the function $t \in[0,1] \rightarrow L_{m(t)}$ belongs to $L^{1}[0,1]$. If $F$ is linear with respect to the first, second and third variables, then

$$
\begin{aligned}
& T_{F, m}\left(\frac{4}{(b-a)^{2}}\left|\frac{1}{b-a} \int_{a}^{b} f(x) d x-f\left(\frac{a+b}{2}\right)\right|,\left|f^{\prime \prime}(a)\right|+\left|f^{\prime \prime}(b)\right|,\left|f^{\prime \prime}(a)\right|+\left|f^{\prime \prime}(b)\right|\right) \\
& +\int_{0}^{1} L_{m(t)} d t \leq 0
\end{aligned}
$$

where

$$
m(t)= \begin{cases}t^{2}, & t \in\left[0, \frac{1}{2}\right] \\ (1-t)^{2}, & t \in\left(\frac{1}{2}, 1\right] .\end{cases}
$$

Proof. Since $\left|f^{\prime \prime}\right|$ is $F$-convex, we have

$$
F\left(\left|f^{\prime \prime}(t a+(1-t) b)\right|,\left|f^{\prime \prime}(a)\right|,\left|f^{\prime \prime}(b)\right|, t\right) \leq 0, t \in[0,1]
$$

and

$$
F\left(\left|f^{\prime \prime}(t b+(1-t) a)\right|,\left|f^{\prime \prime}(b)\right|,\left|f^{\prime \prime}(a)\right|, t\right) \leq 0, t \in[0,1] .
$$

Using the linearity of $F$, we obtain

$$
\begin{aligned}
& F\left(\left|f^{\prime \prime}(t a+(1-t) b)\right|+\left|f^{\prime \prime}(t b+(1-t) a)\right|,\right. \\
& \left.\left|f^{\prime \prime}(b)\right|+\left|f^{\prime \prime}(a)\right|,\left|f^{\prime \prime}(b)\right|+\left|f^{\prime \prime}(a)\right|, t\right) \leq 0,
\end{aligned}
$$

for $t \in[0,1]$. Multiplying this inequality by $m(t)$ and using axiom (A3), we get

$$
\begin{aligned}
& F\left(m(t)\left[\left|f^{\prime \prime}(t a+(1-t) b)\right|+\left|f^{\prime \prime}(t b+(1-t) a)\right|\right], m(t)\left[\left|f^{\prime \prime}(b)\right|+\left|f^{\prime \prime}(a)\right|\right],\right. \\
& \left.m(t)\left[\left|f^{\prime \prime}(b)\right|+\left|f^{\prime \prime}(a)\right|\right], t\right)+L_{m(t)} \leq 0,
\end{aligned}
$$

for $t \in[0,1]$. Integrating over $[0,1]$ with respect to the variable $t$ and using axiom (A2), we obtain

$$
\begin{aligned}
& T_{F, m}\left(\int_{0}^{1} m(t)\left[\left|f^{\prime \prime}(t a+(1-t) b)\right|+\left|f^{\prime \prime}(t b+(1-t) a)\right|\right] d t,\right. \\
& \left.\left|f^{\prime \prime}(b)\right|+\left|f^{\prime \prime}(a)\right|,\left|f^{\prime \prime}(b)\right|+\left|f^{\prime \prime}(a)\right|\right)+\int_{0}^{1} L_{m(t)} d t \leq 0,
\end{aligned}
$$

for $t \in[0,1]$. On the other hand, using Lemma 3, we have

$$
\begin{aligned}
& \frac{4}{(b-a)^{2}}\left|\frac{1}{b-a} \int_{a}^{b} f(x) d x-f\left(\frac{a+b}{2}\right)\right| \\
\leq & \int_{0}^{1} m(t)\left[\left|f^{\prime \prime}(t a+(1-t) b)\right|+\left|f^{\prime \prime}(t b+(1-t) a)\right|\right] d t .
\end{aligned}
$$


Since $T_{F, m}$ is nondecreasing with respect to the first variable, we get

$$
\begin{aligned}
& T_{F, m}\left(\frac{4}{(b-a)^{2}}\left|\frac{1}{b-a} \int_{a}^{b} f(x) d x-f\left(\frac{a+b}{2}\right)\right|,\right. \\
& \left.\left|f^{\prime \prime}(a)\right|+\left|f^{\prime \prime}(b)\right|,\left|f^{\prime \prime}(a)\right|+\left|f^{\prime \prime}(b)\right|\right)+\int_{0}^{1} L_{m(t)} d t \leq 0 .
\end{aligned}
$$

This completes the proof.

Corollary 13. Let $f: I^{\circ} \subset \mathbb{R} \rightarrow \mathbb{R}$ be twice differentiable function on $I^{\circ}, a, b \in I^{\circ}$ with $a<b$. Suppose that the function $\left|f^{\prime \prime}\right|$ is $\varepsilon-$ convex on $[a, b], \varepsilon \geq 0$. Then we have

$$
\left|\frac{1}{b-a} \int_{a}^{b} f(x) d x-f\left(\frac{a+b}{2}\right)\right| \leq \frac{(b-a)^{2}}{4}\left[\frac{\left|f^{\prime \prime}(a)\right|+\left|f^{\prime \prime}(b)\right|}{12}+\frac{\varepsilon}{12}\right] .
$$

Proof. Using (1.4) with $w(t)=m(t)$, we obtain

$$
\begin{aligned}
\int_{0}^{1} L_{m(t)} d t & =\varepsilon \int_{0}^{1}(1-m(t)) d t \\
& =\varepsilon\left(\int_{0}^{1 / 2}\left(1-t^{2}\right) d t+\int_{1 / 2}^{1}\left(1-(1-t)^{2}\right) d t\right) \\
& =\frac{11}{12} \varepsilon .
\end{aligned}
$$

From (1.3) with $w(t)=m(t)$, we get

$$
\begin{aligned}
T_{F, w}\left(u_{1}, u_{2}, u_{3}\right) & =u_{1}-\left(\int_{0}^{1} t m(t) d t\right) u_{2}-\left(\int_{0}^{1}(1-t) m(t) d t\right) u_{3}-\varepsilon \\
& =u_{1}-\frac{u_{2}+u_{3}}{24}-\varepsilon
\end{aligned}
$$

for $u_{1}, u_{2}, u_{3} \in \mathbb{R}$. Hence,

$$
\begin{aligned}
& T_{F, m}\left(\frac{4}{(b-a)^{2}}\left|\frac{1}{b-a} \int_{a}^{b} f(x) d x-f\left(\frac{a+b}{2}\right)\right|,\right. \\
& \left.\left|f^{\prime \prime}(b)\right|+\left|f^{\prime \prime}(a)\right|,\left|f^{\prime \prime}(b)\right|+\left|f^{\prime \prime}(a)\right|\right) \\
& =\frac{4}{(b-a)^{2}}\left|\frac{1}{b-a} \int_{a}^{b} f(x) d x-f\left(\frac{a+b}{2}\right)\right|-\frac{\left|f^{\prime \prime}(a)\right|+\left|f^{\prime \prime}(b)\right|}{12}-\varepsilon .
\end{aligned}
$$


Thus, by Theorem 8 , we have

$$
\frac{4}{(b-a)^{2}}\left|\frac{1}{b-a} \int_{a}^{b} f(x) d x-f\left(\frac{a+b}{2}\right)\right|-\frac{\left|f^{\prime \prime}(a)\right|+\left|f^{\prime \prime}(b)\right|}{12}-\varepsilon+\frac{11}{12} \varepsilon \leq 0,
$$

that is

$$
\left|\frac{1}{b-a} \int_{a}^{b} f(x) d x-f\left(\frac{a+b}{2}\right)\right| \leq \frac{(b-a)^{2}}{4}\left[\frac{\left|f^{\prime \prime}(a)\right|+\left|f^{\prime \prime}(b)\right|}{12}+\frac{\varepsilon}{12}\right] .
$$

This completes the proof.

Remark 6. If we taking $\varepsilon=0$ in Corollary 13 , then $\left|f^{\prime \prime}\right|$ is convex and we have the inequality

$$
\left|\frac{1}{b-a} \int_{a}^{b} f(x) d x-f\left(\frac{a+b}{2}\right)\right| \leq \frac{(b-a)^{2}}{24}\left[\frac{\left|f^{\prime \prime}(a)\right|+\left|f^{\prime \prime}(b)\right|}{2}\right]
$$

which is given by Sarikaya et al. in [13].

Corollary 14. Let $f: I^{\circ} \subset \mathbb{R} \rightarrow \mathbb{R}$ be twice differentiable function on $I^{\circ}, a, b \in I^{\circ}$ with $a<b$. Suppose that the function $\left|f^{\prime \prime}\right|$ is $\alpha$-convex on $[a, b], \alpha \in(0,1]$. Then we have

$$
\left|\frac{1}{b-a} \int_{a}^{b} f(x) d x-f\left(\frac{a+b}{2}\right)\right| \leq \frac{(b-a)^{2}}{24}\left[\frac{\left|f^{\prime \prime}(a)\right|+\left|f^{\prime \prime}(b)\right|}{2}\right] .
$$

Proof. From (1.6) with $w(t)=m(t)$, we have

$$
\begin{aligned}
T_{F, w}\left(u_{1}, u_{2}, u_{3}\right)= & u_{1}-\left(\int_{0}^{1} t^{\alpha} m(t) d t\right) u_{2}-\left(\int_{0}^{1}\left(1-t^{\alpha}\right) m(t) d t\right) u_{3} \\
= & u_{1}-\frac{1}{(\alpha+1)(\alpha+2)}\left(\frac{2}{\alpha+3}-\frac{1}{2^{\alpha+1}}\right) u_{2} \\
& -\left[\frac{1}{12}-\frac{1}{(\alpha+1)(\alpha+2)}\left(\frac{2}{\alpha+3}-\frac{1}{2^{\alpha+1}}\right)\right] u_{3}
\end{aligned}
$$

for $u_{1}, u_{2}, u_{3} \in \mathbb{R}$. It follows that

$$
\begin{aligned}
& T_{F, w}\left(\frac{4}{(b-a)^{2}}\left|\frac{1}{b-a} \int_{a}^{b} f(x) d x-f\left(\frac{a+b}{2}\right)\right|,\right. \\
& \left.\left|f^{\prime \prime}(b)\right|+\left|f^{\prime \prime}(a)\right|,\left|f^{\prime \prime}(b)\right|+\left|f^{\prime \prime}(a)\right|\right) \\
= & \frac{4}{(b-a)^{2}}\left|\frac{1}{b-a} \int_{a}^{b} f(x) d x-f\left(\frac{a+b}{2}\right)\right|
\end{aligned}
$$




$$
\begin{aligned}
& -\frac{1}{(\alpha+1)(\alpha+2)}\left(\frac{2}{\alpha+3}-\frac{1}{2^{\alpha+1}}\right)\left[\left|f^{\prime \prime}(b)\right|+\left|f^{\prime \prime}(a)\right|\right] \\
& -\left[\frac{1}{12}-\frac{1}{(\alpha+1)(\alpha+2)}\left(\frac{2}{\alpha+3}-\frac{1}{2^{\alpha+1}}\right)\right]\left[\left|f^{\prime \prime}(b)\right|+\left|f^{\prime \prime}(a)\right|\right] \\
= & \frac{4}{(b-a)^{2}}\left|\frac{1}{b-a} \int_{a}^{b} f(x) d x-f\left(\frac{a+b}{2}\right)\right|-\frac{\left|f^{\prime \prime}(b)\right|+\left|f^{\prime \prime}(a)\right|}{12} .
\end{aligned}
$$

which completes the proof.

Corollary 15. Let $f: I^{\circ} \subset \mathbb{R} \rightarrow \mathbb{R}$ be twice differentiable function on $I^{\circ}, a, b \in I^{\circ}$ with $a<b$. Suppose that the function $\left|f^{\prime \prime}\right|$ is $h$-convex on $[a, b]$. Then we have

$$
\begin{aligned}
& \left|\frac{f(a)+f(b)}{2}-\frac{1}{b-a} \int_{a}^{b} f(x) d x\right| \\
\leq & (b-a)^{2}\left(\int_{0}^{1} h(t) t(1-t) d t\right)\left[\frac{\left|f^{\prime \prime}(a)\right|+\left|f^{\prime \prime}(b)\right|}{2}\right] .
\end{aligned}
$$

Proof. From (1.8) with $w(t)=m(t)$, we have

$$
\begin{aligned}
T_{F, w}\left(u_{1}, u_{2}, u_{3}\right) & =u_{1}-\left(\int_{0}^{1} h(t) m(t) d t\right) u_{2}-\left(\int_{0}^{1} h(1-t) m(t) d t\right) u_{3} \\
& =u_{1}-\left(\int_{0}^{1} h(t) m(t) d t\right) u_{2}-\left(\int_{0}^{1} h(t) m(1-t) d t\right) u_{3} \\
& =u_{1}-\left(\int_{0}^{1} h(t) m(t) d t\right)\left(u_{2}+u_{3}\right)
\end{aligned}
$$

for $u_{1}, u_{2}, u_{3} \in \mathbb{R}$. Then, by Theorem 8 ,

$$
\begin{aligned}
& T_{F, w}\left(\frac{4}{(b-a)^{2}}\left|\frac{1}{b-a} \int_{a}^{b} f(x) d x-f\left(\frac{a+b}{2}\right)\right|,\right. \\
& \left.\left|f^{\prime \prime}(b)\right|+\left|f^{\prime \prime}(a)\right|,\left|f^{\prime \prime}(b)\right|+\left|f^{\prime \prime}(a)\right|\right) \\
& =\frac{4}{(b-a)^{2}}\left|\frac{1}{b-a} \int_{a}^{b} f(x) d x-f\left(\frac{a+b}{2}\right)\right|
\end{aligned}
$$




$$
-2\left(\int_{0}^{1} h(t) m(t) d t\right)\left[\left|f^{\prime \prime}(a)\right|+\left|f^{\prime \prime}(b)\right|\right] \leq 0
$$

that is,

$\left|\frac{1}{b-a} \int_{a}^{b} f(x) d x-f\left(\frac{a+b}{2}\right)\right| \leq(b-a)^{2}\left(\int_{0}^{1} h(t) m(t) d t\right)\left[\frac{\left|f^{\prime \prime}(a)\right|+\left|f^{\prime \prime}(b)\right|}{2}\right]$

which completes the proof.

\section{REFERENCES}

[1] B. Defnetti, "Sulla strati cazioni convesse." Ann. Math. Pura. Appl., vol. 30, pp. 173-183, 1949.

[2] H. D.H. and S. Ulam, "Approximately convex functions," Proc. Amer. Math. Soc., vol. 3, pp. 821-828, 1952.

[3] S. S. Dragomir and R. P. Agarwal, "Two inequalities for differentiable mappings and applications to special means of real numbers and to trapezoidal formula," Applied Mathematics Letters, vol. 11, no. 5, pp. 91-95, 1998, doi: 10.1016/S0893-9659(98)00086-X.

[4] S. S. Dragomir and C. Pearce, "Selected topics on hermite-hadamard inequalities and applications," 2003.

[5] H. Hudzik and L. Maligranda, "Some remarks ons-convex functions," Aequationes Mathematicae, vol. 48, no. 1, pp. 100-111, 1994, doi: 10.1007/BF01837981.

[6] U. S. Kirmaci, "Inequalities for differentiable mappings and applications to special means of real numbers and to midpoint formula," Applied Mathematics and Computation, vol. 147, no. 1, pp. 137-146, 2004, doi: 10.1016/S0096-3003(02)00657-4.

[7] O. L. Mangasarian, "Pseudo-convex functions," in Stochastic Optimization Models in Finance. Elsevier, 1975, pp. 23-32, doi: 10.1016/B978-0-12-780850-5.50009-5.

[8] J. E. Peajcariaac and Y. L. Tong, Convex functions, partial orderings, and statistical applications. Academic Press, 1992.

[9] C. E. Pearce and J. Pečarić, "Inequalities for differentiable mappings with application to special means and quadrature formulae," Applied Mathematics Letters, vol. 13, no. 2, pp. 51-55, 2000, doi: 10.1016/S0893-9659(99)00164-0.

[10] B. T. Polyak, "Existence theorems and convergence of minimizing sequences in extremum problems with restrictions," Soviet Math. Dokl., vol. 7, pp. 72-75, 1966.

[11] B. Samet, "On an implicit convexity concept and some integral inequalities," Journal of Inequalities and Applications, vol. 2016, no. 1, p. 308, 2016, doi: 10.1186/s13660-016-1253-3.

[12] M. Z. Sarikaya, A. Saglam, and H. Yildirim, "On some hadamard-type inequalities for h-convex functions," J. Math. Inequal, vol. 2, no. 3, pp. 335-341, 2008, doi: 10.7153/jmi-02-30.

[13] M. Z. Sarikaya, A. Saglam, and H. Yildirim, "New inequalities of hermite-hadamard type for functions whose second derivatives absolute values are convex and quasi-convex," International Journal of Open Problems in Computer Science and Mathematics, vol. 238, no. 1390, pp. 1-14, 2012, doi: 10.12816/0006114.

[14] S. Varošanec, "On h-convexity," Journal of Mathematical Analysis and Applications, vol. 326, no. 1, pp. 303-311, 2007, doi: 10.1016/j.jmaa.2006.02.086. 
Authors' addresses

\section{H. Budak}

Düzce University, Faculty of Science and Arts, Department of Mathematics Düzce, Turkey

E-mail address: hsyn.budak@gmail.com

T. Tunç

Düzce University, Faculty of Science and Arts, Department of Mathematics Düzce, Turkey

E-mail address: tubatunc03@gmail.com

\section{Z. Sarikaya}

Düzce University, Faculty of Science and Arts, Department of Mathematics Düzce, Turkey

E-mail address: sarikayamz@gmail.com 